\title{
Naturalistic weight reduction efforts predicted weight gain and onset of obesity in adolescent girls
}

Stice E, Cameron RP, Killen JD, et al. Naturalistic weight-reduction efforts prospectively predict growth in relative weight and onset of obesity among female adolescents.J Consult Clin Psychol 1999 Dec;67:967-74.

QUESTION: Are naturalistic dieting (weight control in the natural environment without formal treatment), exercise, radical weight loss efforts, and binge eating associated with growth in relative weight and onset of obesity in adolescent girls?

Design

Prospective cohort study with 4 years of follow up.

Setting

3 high schools in northern California, USA.

\section{Participants}

692 girls (mean age 15 y, $45 \%$ white) who were grade 9 students. Girls who were pregnant were excluded.

\section{Assessment of risk factors}

Dietary restraint, self labelled dieting, exercise for weight loss, incidental exercise (exercise for purposes other than intentional weight control) that might affect adiposity, fasting for weight loss, use of appetite suppressants or laxatives, vomiting for weight control, binge eating, and stage of pubertal development (Tanner stage) were assessed by student self report or structured clinical interviews. Body mass index (BMI) was measured by trained research staff.

\section{Main outcome measures}

Growth in relative weight and, after excluding initially obese girls ( $16 \%$ of the initial cohort), onset of obesity.

\section{Main results}

Analyses were adjusted for baseline BMI and sexual maturation level. Baseline BMI was not related to subsequent growth in relative weight. Baseline incidental exercise, appetite suppressant or laxative use, vomiting for weight control, and binge eating predicted relative weight gain over the 4 year study period $(\mathrm{p}<0.05)$. Dietary restraint, self labelled dieting, and exercise for weight control predicted onset of obesity over time. For each unit of increase on the restraint scale, there was a 192\% increase in the hazard for onset of obesity (hazard ratio [HR] 2.92, CI 1.74 to $4.89, \mathrm{p}<0.001$ ). The hazard for onset of obesity was $324 \%$ greater for self labelled dieters than for non-dieters (HR 3.24, CI 1.53 to $6.85, \mathrm{p}<0.01$ ), and for each unit increase on the exercise scale, there was a $25 \%$ increase in the hazard for onset of obesity over the study period (HR 1.25, CI 1.08 to $1.45, \mathrm{p}<0.01$ ).

\section{Conclusions}

Incidental exercise, appetite suppressant or laxative use, vomiting, and binge eating predicted relative weight gain in adolescent girls. Dietary restraint, self labelled dieting, and exercise for weight control predicted onset of obesity.

\section{COMMENTARY}

Research on the development of adolescent obesity is limited. This prospective study by Stice $e t a l$ is one of the few studies that examine the relationship between self employed weight control efforts and weight change and obesity. The findings show that dieting and weight loss efforts predicted increases rather than decreases in relative weight and an increased risk of onset of obesity. The findings have credibility within the context of a rising prevalence of obesity among adult women in the United States despite a dieting prevalence $>50 \%{ }^{1}$

This study is methodologically strong, with analyses controlling for initial BMI and sexual maturity level. The participants were predominantly white and, therefore, the results may not be applicable to ethnic groups with particular vulnerability to obesity. The use of self definition for dieting and the lack of specification of the type and duration of exercise did not allow the researchers to know if participants were actually reducing calories or exercising by standard definitions. Previous studies have shown that people may attribute activities, even those not related to food, as "dieting" activities. ${ }^{2}$

The results of this study increase our understanding of the potential effects of "generic dieting", exercise for weight control, and radical weight loss activities on weight and onset of obesity. The notion that such reported activities may be markers for adolescents who may become obese and who may benefit from early intervention is relevant to clinicians. These findings reinforce the need for clinicians to identify adolescents at high risk, assess the meaning of dieting and exercise for weight control, and provide tailored information and interventions to prevent weight gain. The findings of this study are relevant to nurses in the areas of paediatrics, public health, and family, school, and women's health. These clinicians are in a position to initiate obesity risk assessments and primary prevention programmes for adolescents. It is important that these prevention programmes be accompanied by evaluations to assess their effectiveness. Janet Allan, RN, PhD Dean, School of Nursing The University of Texas Health Science Center at San Antonio San Antonio, Texas, USA

1 Dalton S. Overweight and weight management: the health professional's guide to understanding and practice. Gaithersburg, MD Aspen Publishers, 1997.

2 Tyler DO, Allan JD, Alcozer FR. Weight loss methods used by African American and Euro-American women. Res Nurs Health 1997;20:413-23. 